\title{
Narrativa
}

narrativa

Nuova serie

$41 \mid 2019$

Narrativa italiana degli anni Duemila: cartografie e percorsi

\section{Nella BIANCHI BENSIMON (a cura di), Battista Fregoso, Anteros sive contra amorem}

\section{Michel Pretalli}

\section{(2) OpenEdition \\ Journals}

Edizione digitale

URL: https://journals.openedition.org/narrativa/532

DOI: $10.4000 /$ narrativa.532

ISSN: 2804-1224

Editore

Presses universitaires de Paris Nanterre

\section{Edizione cartacea}

Data di pubblicazione: 1 décembre 2019

Paginazione: 169-170

ISBN: 978-2-84016-350-3

ISSN: 1166-3243

\section{Notizia bibliografica digitale}

Michel Pretalli, «Nella BiAnCH BEnsimon (a cura di), Battista Fregoso, Anteros sive contra amorem», Narrativa [Online], 41 | 2019, online dal 01 novembre 2021, consultato il 08 décembre 2021. URL: http://journals.openedition.org/narrativa/532 ; DOI: https://doi.org/10.4000/narrativa.532

Questo documento è stato generato automaticamente il 8 décembre 2021.

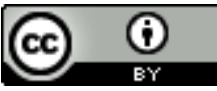

Narrativa est mise à disposition selon les termes de la Licence Creative Commons Attribution 4.0 International. 


\title{
Nella BIANCHI BENSIMON (a cura di), Battista Fregoso, Anteros sive contra amorem
}

\author{
Michel Pretalli
}

\section{NOTIZIA}

Nella BIANCHI BENSIMON (a cura di), Battista Fregoso, Anteros sive contra amorem, Roma, Vecchiarelli Editore,2018, pp. 356.

1 Con l'edizione critica e il ricco commento dell'Anteros sive contra amorem, dialogo del letterato e doge genovese Battista Fregoso (1452-1504), pubblicato nel 1496 a Milano da Leonardo Pachel, Nella Bianchi Bensimon offre al pubblico un testo di notevole importanza nella cultura e nella tradizione letteraria del Rinascimento ma fino ad ora difficilmente reperibile. Di stampo ciceroniano e redatto in volgare, l'Anteros si colloca agli inizi della florida tradizione dialogica rinascimentale, la quale raccolse l'eredità antica per alimentare una produzione letteraria che spazia in quasi tutti i campi dello scibile. Il volume, edito da Vecchiarelli (2018), comprende una ricca introduzione (pp. 7-122) seguita da un'appendice del prezioso inventario parziale (1425) della biblioteca di Tommaso Campofregoso, antenato di Battista, da una nota al testo in cui vengono precisati sia la copia di riferimento usata (Biblioteca Mazarine di Parigi, Inc. 862), sia la lista degli esemplari noti e i criteri di trascrizione con tavola dei refusi. Segue il testo commentato dell'Anteros: "Platinus in Anterota illustris Baptistae C. Fulgosi ad lectorem"; "Auctor tyronibus Amoris"; Baptistae C. Fulgosi, Anterotis ad splendidum Equitem Ioannem Franciscum Pusterlam. Liber primus. Prologus; "Ragionando el Piatino e Baptista"; "Baptistae Fulgosi Anterotis Liber Secundus"; "Apostrophe ad librum". Completano il volume un Regesto bibliografico e l'indice dei nomi. Nella corposa introduzione, la curatrice, specialista del dialogo umanista in lingua volgare e più particolarmente di Leon Battista Alberti, mostra il posto di rilievo occupato 
dall'Anteros in questa tradizione e mette efficacemente in luce la maniera in cui Fregoso sfrutta la forma dialogica a fini persuasivi. Non si tratta di un dialogo eristico ed euristico, poiché non esiste dubbio che prevarrà la reprobatio amoris difesa dal personaggio di Piatino Piatti, ma di una sorta di disputatio in utramque partem. La presenza di più interlocutori - Battista e Claudio di Savoia oltre a Piatino - consente di evitare la monotonia delle lunghe orationes e di presentare il soggetto trattato da più punti di vista nel quadro di una piacevole discussione conforme ai criteri dell'urbanitas. Il testo si presenta come una sorta di epitome delle conoscenze relative all'amore, questione estremamente complessa e dibattuta nella cultura occidentale. Battista Fregoso abbraccia la tesi antierotica, considerando Anteros non come complementare di Eros ma come il suo opposto: l'amore, infatti, semplice passione umana, viene descritta come un "fattore di alienazione della ratio maschile" che mette in pericolo non solo la salute, mentale e fisica, dell'uomo ma persino l'ordine sociale, e deve perciò essere canalizzato dal coniugio. Per difendere la sua tesi, Piatino fa leva sulla sua vasta erudizione costellando il discorso di citazioni tratte dai testi classici, dalla teologia e dalla medicina medievali, ma anche da Dante, Boccaccio e soprattutto Petrarca. Oltre alla edizione dell'Anteros, che consente al pubblico moderno di godere di un'opera letteraria e scientifica di notevole spessore, Nella Bianchi Bensimon, attraverso la contestualizzazione e l'analisi approfondita del testo, con ricostruzione meticolosa delle fonti, palesa il percorso intellettuale del Fregoso fornendo argomentazioni tanto equilibrate quanto convincenti e aggiunge un tassello importante non solo alla conoscenza delle dinamiche dei primi dialoghi in volgare ma più in generale della tradizione culturale e letteraria del Rinascimento. 